\title{
Expertenstellungnahme zur Substitutionstherapie bei Patienten mit Alpha-1 Antitrypsin-Mangel*
}

\section{Expert Statement for Augmentation Therapy in Patients with Alpha-1 Antitrypsin Deficiency}

Autoren

Institute
T. Köhnlein ${ }^{1}$, K. Schmidt-Scherzer ${ }^{2}$, T. Greulich ${ }^{3}$, R. Bals ${ }^{4}$

Die Institutsangaben sind am Ende des Beitrags gelistet. eingereicht $\quad 25.4 .2014$ akzeptiert nach Revision 5.5.2014

Bibliografie

Dol http://dx.doi.org/ 10.1055/s-0034-1365802 Pneumologie 2014; 68: 492-495 (c) Georg Thieme Verlag KG Stuttgart · New York ISSN 0934-8387

Korrespondenzadresse PD Dr. med. Thomas Köhnlein Klinikum St. Georg gGmbH Robert-Koch-Klinik Nikolai-Rumjanzew-Straße 100 04207 Leipzig

thomas.koehnlein@stgeorg.de

\section{Zusammenfassung \\ $\nabla$}

Die Substitutionstherapie bei Alpha-1 Antitrypsin-Mangel soll das Voranschreiten eines Lungenemphysems verzögern, die Exazerbationshäufigkeit reduzieren und die Lebensqualität der Patienten verbessern. Dieses Expertenstatement fasst die wichtigsten Therapiestudien zur Substitutionstherapie bei Patienten mit schwerem Alpha-1 Antitrypsin-Mangel und Lungenemphysem kurz zusammen. Aus den verfügbaren Studienergebnissen werden Indikationen und Kontraindikationen für eine dauerhafte intravenöse Substitutionstherapie mit humanem Alpha-1 Antitrypsin abgeleitet. Sicherheitsaspekte und die Kontroversen um das Thema werden ausführlich diskutiert.

\section{Einleitung \\ $\nabla$}

Analog zur Substitution bei anderen erblich bedingten Protein-Mangelkrankheiten besteht beim Alpha-1 Antitrypsin-Mangel die Therapieoption einer Substitution mit humanem Alpha-1 Antitrypsin (AAT) [1]. Diese Substitutionstherapie ist in Deutschland und Österreich seit 1989 eine zugelassene Dauertherapie für Patienten mit schwerem Alpha-1 Antitrypsin-Mangel. Die Zulassung gilt für die Therapie von Patienten mit den Genotypen $\mathrm{PI}^{*} \mathrm{ZZ}, \mathrm{PI}^{*} \mathrm{SZ}, \mathrm{PI}^{*} \mathrm{Z}$ (Null) und $\mathrm{PI}^{*}$ (Null-Null), die eine mittelgradig eingeschränkte Lungenfunktion mit einem $\mathrm{FEV}_{1}$ im Bereich von 35 bis $60 \%$ des altersentsprechenden Sollwerts aufweisen. Die Zulassung sieht eine einmal wöchentliche Gabe von $60 \mathrm{mg}$ AAT pro kg Körpergewicht vor. Die Zahl der substituierten Patienten

\footnotetext{
* Diese Expertenstellungnahme wurde auf einem Treffen von Vertretern der Alpha-1 Center aus Deutschland und Österreich am 7.12.2013 entwickelt. Die vollständige Liste der Mitwirkenden befindet sich am Ende der Expertenstellungnahme.
}

\section{Abstract \\ $\nabla$}

Augmentation therapy in Alpha-1 Antitrypsin Deficiency aims to reduce the progression of lung emphysema, to reduce exacerbation frequency, and to improve quality of life. This expert statement briefly summarizes the most important treatment studies performed in patients with $\mathrm{Al}-$ pha-1 Antitrypsin Deficiency and severe lung emphysema. Indications and contraindications for long-term intravenous augmentation therapy with human Alpha-1 Antitrypsin are derived from the available study results. Safety issues and the controversies of this topic are discussed in detail.

liegt in Deutschland bei schätzungsweise 900, in Österreich bei 80 bis 90 .

Beobachtungen an Trägern des Genotyps $\mathrm{PI}^{*} \mathrm{SZ}$ zeigten, dass ab einer AAT Serum-Konzentration von weniger als $0,5 \mathrm{~g} / \mathrm{l}(11 \mu \mathrm{mol} / \mathrm{l})$ mit der Entwicklung eines Lungenemphysems zu rechnen ist [2]. Diese Konzentration gilt seitdem als „protektive Schwelle“, d. h. als Mindest-Konzentration von AAT ohne wesentlich erhöhtes Risiko für die Entwicklung eines Lungenemphysems [3]. Durch intravenöse Applikation von humanem AAT kann die AAT-Konzentration im Serum und im epithelialen Flüssigkeitsfilm der Alveolen über einen Zeitraum von einigen Tagen über die protektive Schwelle angehoben werden [4].

Das vorliegende Expertenstatement referiert vor dem Hintergrund der verfügbaren Therapie-Studien und in Übereinstimmung mit den gültigen Empfehlungen der Fachgesellschaften $[5,6]$ die Indikation zur dauerhaften Substitutionstherapie. Selbstverständlich soll durch die hier vorgestellte Empfehlung die ärztliche Therapiefreiheit in keiner Weise eingeschränkt werden. Die individuelle Situation jedes einzelnen Patienten kann ein be- 
gründetes Abweichen jederzeit erforderlich machen. Zu einer ausführlichen Darstellung des Krankheitsbildes Alpha-1 Antitrypsin-Mangel verweisen wir auf die Arbeit von S. Schroth et al., Pneumologie 2009 [7] und auf international publizierte Reviews $[8,9]$.

\section{Ziele der Substitution}

\section{$\nabla$}

Die Substitutionstherapie soll das Voranschreiten eines Lungenemphysems verlangsamen, die Häufigkeit von Exazerbationen reduzieren und die gesundheitsbezogene Lebensqualität des Patienten verbessern.

\section{Wissenschaftlicher Hintergrund \\ $\nabla$}

In zwei randomisierten, kontrollierten, prospektiven Studien (Evidenzgrad A-vgl. Tab.1) konnte mit Hilfe der computertomografisch gemessenen Dichte des Lungengewebes bei regelmäßig AAT-substituierten Patienten ein Trend zu einer geringeren Abnahme der Lungendichte beobachtet werden. Abhängig vom angewandten statistischen Modell wurden in Beobachtungszeiträumen von 2,5 bis 3 Jahren signifikante Unterschiede zwischen AAT-Therapie und Placebo-Behandlung deutlich [10, 11]. Eine dritte, multinationale Studie mit sehr ähnlichem Design und gleichem Dosisregime zeigte ein klares und statistisch signifikantes Signal für eine verminderte Verlustrate der Lungendichte unter intravenöser Substitution. Diese Studie liegt bislang allerdings nur als Abstract vor [12].

Zwei retrospektive Kohorten-Studien (beide Evidenzgrad C) zeigten, dass die Letalität von substituierten Patienten [13] und der jährliche $\mathrm{FEV}_{1}$-Verlust vor allem bei Patienten mit mittelgradig

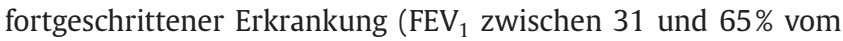
Soll) [14] signifikant gebessert werden können. Ähnliche Beobachtungen werden in einer prospektiven Studie berichtet, in der 287 Patienten nach Beginn einer i.v.-Substitutionstherapie über durchschnittlich 37 Monate nachbeobachtet wurden. Patienten mit einem Ausgangs-FEV ${ }_{1}$ im Bereich von 30 bis $65 \%$ konnten ihren jährlichen $\mathrm{FEV}_{1}$-Verlust gegenüber dem erwarteten Abfall des Atemstoßes etwa halbieren (Evidenzgrad B) [18]. Eine Meta-Analyse aus dem Jahr 2009, in die 1509 Patienten eingeschlossen wurden, zeigte, dass durch Substitutionstherapie der jährliche FEV $_{1}$-Verlust um durchschnittlich $23 \%$ (absolut 13,5 ml/Jahr) reduziert werden kann. Dieser Effekt zeigte sich auch hier am deutlichsten bei Patienten mit einem $\mathrm{FEV}_{1}$-Wert bei Therapiebeginn zwischen 30 und 65\% vom altersentsprechenden Sollwert [15]. Bei Patienten mit einem $\mathrm{FEV}_{1}$ von unter $30 \%$ des Sollwertes lie-

Tab. 1 Definition der Evidenzgrade gemäß der GOLD-Initiative [1].

\begin{tabular}{|ll|}
\hline Evidenzgrad & Definition \\
\hline A & $\begin{array}{l}\text { konsistente Daten von randomisierten, kontrollierten } \\
\text { Studien mit großer Patientenzahl }\end{array}$ \\
\hline B & $\begin{array}{l}\text { randomisierte, kontrollierte Studien mit geringerer } \\
\text { Patientenzahl und gewissen Inkonsistenzen, Meta- } \\
\text { Analysen von randomisierten, kontrollierten Studien }\end{array}$ \\
\hline C & $\begin{array}{l}\text { Ergebnisse aus unkontrollierten oder nicht randomi- } \\
\text { sierten Studien oder aus Beobachtungen }\end{array}$ \\
\hline D & $\begin{array}{l}\text { Stellungnahme anhand der Erfahrung von Experten } \\
\text { ohne eindeutige Studienergebnisse wie bei A-C }\end{array}$ \\
\hline
\end{tabular}

gen keine ausreichenden Daten zur Beurteilung der Effekte einer Substitutionstherapie vor.

Studien zur Exazerbationshäufigkeit vor- bzw. nach Beginn einer Substitutionstherapie gibt es im Rahmen von Patientenbefragungen. In beiden verfügbaren Arbeiten hatte sich die Exazerbationshäufigkeit signifikant verringert (beide Evidenzgrad C) [16,17].

Aus den Erkenntnissen der genannten Untersuchungen sowie aus einer Analyse von Patienten mit raschem Funktionsverlust [18] resultieren in Analogie zu den gültigen nationalen und internationalen Leitlinien $[5,6]$ die folgenden Therapievorschläge.

\section{Indikationen zur i. v.-Substitution mit humanem AAT} $\nabla$

Die Indikationsstellung beruht auf folgenden Kriterien:

1. Es muss ein Alpha-1 Antitrypsin-Mangel mit einem Serumspiegel unter 0,5g/l bzw. $11 \mu \mathrm{mol} / \mathrm{l}$ und ein Lungenemphysem vorliegen. Dabei handelt es sich zumeist um den Genotyp $\mathrm{PI}^{*} \mathrm{ZZ}$, andere Genotypen sind eine Rarität.

2. a) Die Patienten müssen eine Lungenfunktionseinschränkung mit einem Tiffeneau-Wert $\left(\mathrm{FEV}_{1} / \mathrm{FVC}\right)$ von $<0,7$ und einen postbronchodilatatorischen $\mathrm{FEV}_{1}$-Wert von unter $65 \%$ des altersentsprechenden Sollwertes aufweisen. Die Messung muss in einem infektfreien Intervall unter optimierter medikamentöser Dauertherapie erfolgen.

b) Eine Substitution kann auch bei einem postbronchodilatatorischen $\mathrm{FEV}_{1}$-Wert von über $65 \%$ des altersentsprechenden Sollwertes begonnen werden, wenn ein ausgeprägter Abfall des $\mathrm{FEV}_{1}$ von über $50 \mathrm{ml}$ pro Jahr vorliegt (Evidenzgrad B, vgl. COPD-Leitlinie der Deutschen Gesellschaft für Pneumologie und Beatmungsmedizin e.V. [6]). Dieser Abfall ist mit wiederholten Messungen zu dokumentieren, um die bei der Lungenfunktion zwangsläufig vorhandene Messungenauigkeit als Ursache der Änderung der Messwerte auszuschließen.

c) Bei Patienten mit schwerem AAT-Mangel und schwerer Funktionseinschränkung ( $\mathrm{FEV}_{1}<30 \%$ des Sollwertes) kann die Neueinstellung auf die Substitutionstherapie nicht generell empfohlen werden [6], eine laufende Therapie sollte aber auch nach Unterschreiten der $\mathrm{FEV}_{1}$-Grenze von 30\% des Solls nicht automatisch beendet werden [5].

3. Objektive Kriterien für die Beendigung einer laufenden Substitutionstherapie sind nicht verfügbar.

4. Daten zu einer oberen oder unteren Altersgrenze für Substitutionspatienten liegen nicht vor.

\section{Kontraindikationen \\ $\nabla$}

Angesichts der Inaktivierung des AAT durch Zigarettenrauch ist die Substitutionstherapie medizinisch nur dann sinnvoll, wenn kein inhalativer Tabakkonsum vorliegt. Die Patienten sind daher bei jeder ärztlichen Untersuchung auf den notwendigen Verzicht auf Tabakkonsum hinzuweisen. In Zweifelsfällen sollte die Kohlenmonoxid-Konzentration und/oder Cotinin, ein Abbauprodukt von Nikotin, im Blut gemessen werden. Vor Beginn einer Substitutionstherapie ist ggf. eine professionell begleitete Raucherentwöhnung durchzuführen.

Eiweißunverträglichkeiten sowie ein kompletter IgA-Mangel sind weitere Kontraindikationen für eine Substitutionstherapie. Da die Substitution mit AAT zu einer kurzfristigen Erhöhung des Blutvolumens führen kann, ist bei Patienten mit schwerer Herz- 
insuffizienz und bei Patienten mit dem Risiko einer Kreislaufüberlastung besondere Vorsicht geboten.

Andere Erkrankungen, die mutmaßlich einen größeren Einfluss auf die klinische Situation und die Prognose des Patienten als die Folgen des Alpha-1 Antitrypsin-Mangels haben, stellen eine relative Kontraindikation für eine Substitutionstherapie dar.

\section{Sicherheit \\ $\nabla$}

Weltweit sind nur wenige Nebenwirkungen aufgetreten. Beobachtet wurden Fieber, Schüttelfrost und Unwohlsein, die alle nach Änderung der Charge oder Absetzen der Therapie sistierten. Anaphylaktische Reaktionen oder Antikörperbildung gegen Alpha-1 Antitrypsin wurden nur in wenigen Einzelfällen beobachtet [19]. Eine Virusübertragung (Hepatitis B oder C, sowie HIV) wurde nach über 25-jähriger Therapieerfahrung nicht beobachtet. Die Bestimmung einer Hepatitis- und HIV-Serologie vor Substitutionsbeginn ist nicht obligatorisch, jedoch ratsam. Das gleiche gilt für eine Impfung gegen Hepatitis A und B.

Bei klinisch stabilen Patienten kann die Substitution im ambulanten Bereich begonnen und auf Dauer durchgeführt werden.

\section{Kontroversen}

$\nabla$

Die Machbarkeit einer prospektiven, randomisierten klinischen Studie mit „harten“ klinischen Endpunkten wird seit Verfügbarkeit von AAT zur i.v.-Substitution diskutiert. Aufgrund der geringen Anzahl der Patienten bei einer seltenen Lungenerkrankung und der langsamen durchschnittlichen Progredienz des Lungenemphysems sind Studien mit dem Endpunkt Sterblichkeit unrealistisch. Eine Studie mit dem primären Endpunkt FEV $_{1}$-Abfall würde nach einer statistischen Analyse der notwendigen Untersuchungszahlen zur Darstellung signifikanter Ergebnisse pro Arm mindestens 147 Patienten benötigen, um in 4 Jahren Beobachtungszeit einen Unterschied im $\mathrm{FEV}_{1}$-Abfall von $23 \mathrm{ml}$ pro Jahr nachzuweisen [20].

Die Daten der ersten beiden oben erwähnten Densitometrie-Studien $[10,11]$ mit insgesamt 119 Patienten wurden vor kurzem kombiniert und erneut analysiert [21]. Auch die Analyse der gepoolten Daten zeigte einen signifikanten, positiven Effekt hinsichtlich der Entwicklung der Gewebedichte bei Patienten unter dauerhafter Substitution. Die Autoren interpretieren die Abschwächung des Verfalls der Gewebedichte als das entscheidende Surrogat für die Beurteilung der Progredienz eines Lungenemphysems. Ein Cochrane Review von Gøtzsche et al. [22] resümiert, dass aus der Betrachtung der beiden Densitometrie-Studien keine allgemeine Therapieempfehlung abgeleitet werden kann, weil in den beiden zugrunde liegenden Studien der Endpunkt FEV 1 keinen Unterschied zeigte und Sterblichkeit und unerwünschte Effekte der Substitutionstherapie nicht untersucht wurden. Die beiden genannten Densitometrie-Studien waren allerdings nicht für die Untersuchung von $\mathrm{FEV}_{1}$ oder Sterblichkeit konzipiert, sondern der Verlauf der Lungendichte war der primäre Endpunkt, für den die Fallzahlberechnung und Studienkonzeption erfolgte.

Patienten mit akuten Exazerbationen sind besonders gefährdet und erleiden häufig einen Abfall des $\mathrm{FEV}_{1}$. Theoretische Überlegungen legen nahe, dass in dieser Situation der Schutz durch die Substitutionstherapie nicht ausreicht und ein Überschuss an proteolytischen Enzymen in der Lunge besteht. Daten zur Modi- fikation des Substitutionsschemas während einer Exazerbation existieren nicht.

\section{Fazit für die Praxis}

Voraussetzung für eine Substitution ist ein mittelschweres Stadium des Alpha-1 Antitrypsin-Mangels (fast immer Genotyp $\mathrm{PI}^{*} \mathrm{ZZ}$ ). Die genannten Lungenfunktionswerte mit $\mathrm{FEV}_{1}$-Grenzen von $65 \%$ und $30 \%$ vom altersentsprechenden Sollwert wurden aus den Daten der vorliegenden Therapie-Studien abgeleitet. In diesem Lungenfunktionsbereich ist die Indikationsstellung unstrittig. Wenn das $\mathrm{FEV}_{1}$ über $65 \%$ vom Soll beträgt, ist eine abwartende Haltung gerechtfertigt, sofern kein rasch progredienter $\mathrm{FEV}_{1}$-Abfall vorliegt (siehe oben).

Andererseits sind nicht alle Patienten mit einem $\mathrm{FEV}_{1}$ unter $65 \%$ vom Soll automatisch Substitutionskandidaten. Auch in dieser Gruppe gibt es Patienten mit niedriger Exazerbationsrate und, über längere Zeiträume betrachtet, konstanter Lungenfunktion. Solange die Exazerbationsfrequenz niedrig $(<1 / \mathrm{Jahr})$ und die Lungenfunktion stabil ( $<50 \mathrm{ml} \mathrm{FEV}_{1}$-Abfall pro Jahr) bleibt, kommen die Vorteile der Substitution wahrscheinlich nicht zum Tragen.

Wenn die Entscheidung zur Therapie fällt, handelt es sich um eine Dauertherapie. Das am besten untersuchte und in Deutschland und Österreich zugelassene Therapieregime ist die wöchentliche intravenöse Gabe von humanem Alpha-1 Antitrypsin in einer Dosierung von $60 \mathrm{mg}$ pro kg Körpergewicht. Da AAT nur in Dosen von jeweils $1 \mathrm{~g}$ verfügbar ist, ist die applizierte Dosis auf eine volle Grammzahl zu runden. Sieben Tage nach einer Substitution sollte der AAT-Serumspiegel noch oberhalb der protektiven Schwelle von $0,5 \mathrm{~g} / \mathrm{l}$ liegen. Ist das nicht der Fall, soll die applizierte Dosis schrittweise um wöchentlich $1 \mathrm{~g}$ gesteigert werden. In den ersten Wochen nach Beginn einer Substitutionstherapie sollten wiederholte Talspiegel bestimmt werden, um die individuell beste Dosis zu finden. Bei längerfristig stabilem Krankheitsverlauf sollten Talspiegelkontrollen in maximalem Abstand von einem Jahr durchgeführt werden.

\section{Therapieempfehlungen für eine Substitution bei \\ schwerem Alpha-1 Antitrypsin-Mangel}

(Details siehe Text)

Indikationen:

- gesicherte Diagnose Alpha-1 Antitrypsin-Mangel mit einem Alpha-1 Serumspiegel von unter 0,5 g/l bzw. unter $11 \mu \mathrm{mol} / \mathrm{l}$ und

- Tiffeneau-Wert $\left(\mathrm{FEV}_{1} / \mathrm{FVC}\right)$ von $<0,7$ und ein postbronchodilatator $\mathrm{FEV}_{1}$ zwischen $30 \%$ und $65 \%$ des altersentsprechenden Sollwertes

\section{Keine Indikation:}

- Patienten mit normaler Lungenfunktion und Patienten ohne Verschlechterung der Lungenfunktion in der Langzeitbeobachtung

- anhaltender Tabakkonsum

Kontraindikationen:

- Eiweißunverträglichkeiten

- kompletter IgA-Mangel

schwere Herzinsuffizienz 
Vor Beginn einer Substitutionstherapie muss ein IgA-Mangel durch Messung des Gesamt-IgA ausgeschlossen werden. Die Bestimmung einer Hepatitis- und HIV Serologie ist ratsam, das gleiche gilt für eine Impfung gegen Hepatitis A und B. Bei klinisch stabilen Patienten kann die Substitution im ambulanten Bereich begonnen und auf Dauer durchgeführt werden.

Diese Expertenstellungnahme entstand unter der Mitarbeit von: Jost Achenbach, Lostau; Max Apprich, Worms; Wolfgang Gleiber, Frankfurt; Lars Hagmeyer, Solingen; Hubert Hautmann, München; Marc Höffgen, Rheine; Kim Husemann, Gerlingen; János Juhász, Eschwege; Jutta Kappes, Heidelberg; Clemens Kelbel, Dortmund; Klaus Kenn, Schönau/Königssee; Verena Knipel, Köln; Heiko Knoop, Bochum; Marc Lepiorz, Donaustauf; Vera Leucht, Coswig; Joachim Lorenz, Lüdenscheid; Wolfram Meschede, Freiburg; Ernst Wilhelm Müller, Borstel/Lübeck; Astrid Pfitzer, Sonneberg; Angelika Reißig, Jena; Rainer Schädlich, Straelen; Bernhard Schmidt, Münster; Konrad Schulz, Bad Reichenhall; Dirk Skowasch, Bonn; Ulrich Steinhauser, Sinsheim; Michael Steinheimer, Fürth; Heinz Steveling, Essen; Hubert Trötschler, Freiburg; Bernardo Vitiello, Augsburg; Christian Volk, Hamburg; Walter Vorderstraße, Weyhe; Manfred Wagner, Nürnberg; Norbert Weber, München-Gauting; Sabina Wehgartner-Winkler, Augsburg; Andreas Wilke, Berlin; Horst Wittstruck, Bad-Dürrheim.

\section{Interessenkonflikt}

$\nabla$

T. Köhnlein erhielt Reisekostenunterstützung zum Besuch wissenschaftlicher Kongresse und Referenten- bzw. Beraterhonorare durch die Firmen Grifols, Chiesi und CLS Behring. K. SchmidtScherzer erhielt Reisekostenunterstützung zum Besuch wissenschaftlicher Kongresse und Referentenhonorare durch die Firma Chiesi. T. Greulich erhielt Reisekostenunterstützung zum Besuch wissenschaftlicher Kongresse und Referenten- bzw. Beraterhonorare und Unterstützung für Forschungsprojekte und wissenschaftliche Mitarbeiter durch die Firma Grifols. R. Bals erhielt Reisekostenunterstützung zum Besuch wissenschaftlicher Kongresse und Referenten- bzw. Beraterhonorare durch die Firma Grifols.

\footnotetext{
Institute

${ }^{1}$ Klinikum St. Georg gGmbH Leipzig, Robert-Koch-Klinik

${ }^{2}$ Wilhelminenspital Wien, 2. Medizinische Abteilung/Lungenabteilung

${ }^{3}$ Universitätsklinikum Marburg, Klinik für Innere Medizin, Schwerpunkt Pneumologie

${ }^{4}$ Universitätsklinikum des Saarlandes, Innere Medizin V - Pneumologie,

Allergologie, Beatmungs- und Umweltmedizin, Homburg/Saar
}

\section{Literatur}

1 Global Initiative for Chronic Obstructive Lung Disease (GOLD). Global strategy for the diagnosis, management and prevention of COPD. 2013: http: //www.goldcopd.org

2 Ferrarotti I, Thun GA, Zorzetto M et al. Serum levels and genotype distribution of alpha1-antitrypsin in the general population. Thorax 2012; 67: 669-674
3 Crystal RG. Alpha 1-antitrypsin deficiency, emphysema, and liver disease. Genetic basis and strategies for therapy. J Clin Invest 1990; 85: $1343-1352$

4 Stoller JK, Aboussouan LS. Alpha1-Antitrypsin deficiency: intravenous augmentation therapy: current understanding. Thorax 2004; 59: $708-712$

5 American Thoracic Society/European Respiratory Society Statement: Standards for the diagnosis and management of individuals with Alpha-1 Antitrypsin Deficiency. Am J Respir Crit Care Med 2003; 168: $818-900$

6 Vogelmeier C, Buhl R, Criée CP et al. Leitlinie der Deutschen Atemwegsliga und der Deutschen Gesellschaft für Pneumologie und Beatmungsmedizin zur Diagnostik und Therapie von Patienten mit chronisch obstruktiver Bronchitis und Lungenemphysem (COPD). Pneumologie 2007; 61: e1-e40

7 Schroth S, Koczulla R, Herr C et al. Alpha-1-antitrypsin Magel: Diagnose und Therapie der pulmonalen Erkrankung. Pneumologie 2009; 63: $335-341$

8 Köhnlein T, Welte T. Alpha-1 Antitrypsin Deficiency: Pathogenesis, clinical presentation, diagnosis, and treatment. Am J Med 2008; 121: 3 9

9 Stoller JK, Aboussouan LS. Alpha1-antitrypsin deficiency. Lancet 2005; 365: 2225-2236

10 Dirksen A, Dijkman JH, Madsen F et al. A randomized clinical trial of alpha(1)-antitrypsin augmentation therapy. Am J Respir Crit Care Med 1999; 160: 1468 - 1472

11 Dirksen A, Piitulainen E, Parr DG et al. Exploring the role of CT densitometry: a randomised study of augmentation therapy in alpha1-antitrypsin deficiency. Eur Respir J 2009; 33: 1345 - 1353

12 Chapman KR et al. IV Alpha1 Antitrypsin (A1AT) preserves lung density in homozygous Alpha1 Antitrypsin Deficiency (A1ATD); a randomized, placebo-controlled trial. Am J Respir Crit Care Med 2013; 187: A6069

13 The Alpha-1-Antitrypsin Deficiency Registry Study Group. Survival and $\mathrm{FEV}_{1}$ decline in individuals with severe deficiency of alpha1-antitrypsin. Am J Respir Crit Care Med 1998; 158: 49-59

14 Seersholm N, Wencker $M$, Banik $N$ et al. Does alpha1-antitrypsin augmentation therapy slow the annual decline in $\mathrm{FEV}_{1}$ in patients with severe hereditary alpha1-antitrypsin deficiency? Eur Respir J 1997; 10: $2260-2263$

15 Chapman KR, Stockley RA, Dawkins C et al. Augmentation therapy for alpha1 antitrypsin deficiency: a meta-analysis. COPD 2009; 6: $177-$ 184

16 Köhnlein T, Janciauskiene S, Welte T. Diagnostic delay and clinical modifiers in alpha-1 antitrypsin deficiency. Ther Adv Respir Dis 2010; 4 : $279-287$

17 Lieberman J. Augmentation therapy reduces frequency of lung infections in antitrypsin deficiency: a new hypothesis with supporting data. Chest 2000; 118: 1480-1485

18 Wencker M, Fuhrmann B, Banik $N$ et al. Longitudinal follow-up of patients with alpha(1)-protease inhibitor deficiency before and during therapy with IV alpha(1)-protease inhibitor. Chest 2001; 119: $737-$ 744

19 Meyer FJ, Wencker $M$, Teschler $H$ et al. Acute allergic reaction and demonstration of specific IgE antibodies against alpha-1-protease inhibitor. Eur Respir J 1998; 12: 996 -997

20 Schluchter MD, Stoller JK, Barker AF et al. Feasibility of a Clinical Trial of Augmentation Therapy for alpha(1)-Antitrypsin Deficiency. Am J Respir Crit Care Med 2000; 161: 796-801

21 Stockley RA, Parr DG, Piitulainen E et al. Therapeutic efficacy of alpha1 antitrypsin augmentation therapy on the loss of lung tissue: an integrated analysis of 2 randomised clinical trials using computed tomography densitometry. Respir Res 2010; 11: 136

22 Gøtzsche PC, Johansen HK. Intravenous alpha-1 antitrypsin augmentation therapy for treating patients with alpha- 1 antitrypsin deficiency and lung disease. Cochrane Database Syst Rev 2010; 7: CD007851 